\section{La niche \\ hématopoïétique \\ de la drosophile}

$>$ Le maintien et la fonction des cellules souches qui assurent le renouvellement des tissus sont dépendants du microenvironnement de ces cellules, désigné par le terme «niche». Chez les mammifères, plusieurs voies de signalisation ont été impliquées dans les communications entre les cellules souches hématopoḯtiques et leur niche. Nos connaissances de ces communications restent cependant fragmentaires. La découverte chez la drosophile d'une niche hématopoïétique, le posterior signaling center (PSC), a ouvert de nouvelles possibilités d'études génétiques. Le nombre des cellules du PSC est déterminant pour l'homéostasie entre progéniteurs hématopoïétiques et cellules différenciées. Le décryptage d'une cascade de signalisation contrôlant cette taille a établi de nouveaux parallèles entre la drosophile et les mammifères, et ouvert de nouvelles perspectives d'étude chez l'homme. <

\section{La niche hématopoiétique des mammifères}

Les cellules souches jouent un rôle essentiel pour le maintien de l'homéostasie des tissus adultes. L'utilisation des cellules souches, un des espoirs de la médecine régénératrice, nécessite d'identifier et de caractériser les mécanismes moléculaires impliqués dans leur autorenouvellement, leur prolifération et leur différenciation. Il est maintenant acquis que le microenvironnement cellulaire, appelé niche, joue un rôle essentiel dans le contrôle de ces processus. Chez l'homme adulte, les cellules souches hématopoḯtiques (CSH) résident dans la moelle osseuse où elles prolifèrent et se différencient en cellules des différents lignages sanguins : érythrocytaire, myéloïde, lymphoïde et mégacaryocytaire. Deux compartiments majeurs de la niche hématopoïétique sont la niche endostéale (proche de l'os) et la niche vasculaire, contenant différents types de cellules stromales sécrétant des facteurs solubles dans l'environnement des

\section{Un modèle d'étude in vivo du microenvironnement contrôlant les cellules souches hématopoïétiques}

Ismaël Morin-Poulard, Isabelle Louradour, Nathalie Vanzo, Michèle Crozatier

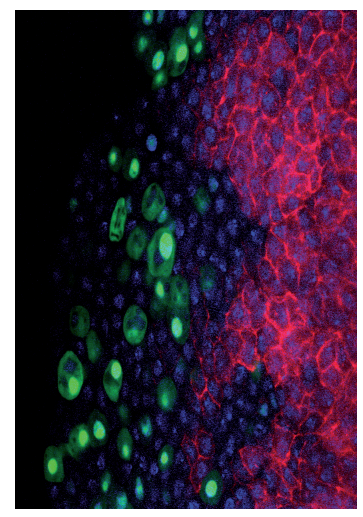

Centre de biologie du développement, UMR 5547 CNRS/UPS, Toulouse III, 118, route de Narbonne, 31062 Toulouse Cedex 9, France. I. Morin-Poulard et I. Louradour ont contribué de façon équivalente à cet article. michele.crozatier-borde@univ$\underline{\text { tlse3.fr }}$

CSH [1-6] (Figure 1). Cependant, bien que le concept de niche ait été proposé dès 1978 [7], les relations fonctionnelles entre niches et CSH restent mal connues. La découverte d'une «niche hématopoïétique» chez la drosophile ouvre de nouvelles possibilités pour étudier in vivo comment le microenvironnement contrôlant l'autorenouvellement et la différenciation des progéniteurs hématopoiétiques est lui-même régulé.

\section{Avantages du modèle drosophile pour des études in vivo}

La majorité des gènes qui régulent l'hématopoïèse sont conservés au cours de l'évolution, de la drosophile aux vertébrés [8]. La redondance fonctionnelle entre gènes/protéines d'une même famille étant beaucoup moins importante chez la drosophile, ce modèle facilite les analyses fonctionnelles in vivo [9]. La drosophile présente aussi un temps de génération court et offre une panoplie de techniques génétiques sophistiquées. Enfin, alors que chez les vertébrés l'hématopoïèse adulte se situe dans la moelle osseuse, elle a lieu chez la drosophile dans un organe facilement accessible et manipulable in vivo : la glande lymphatique.

\section{L’hématopoïèse chez la drosophile}

L'hématopoï̀se de la drosophile, à l'origine des cellules sanguines (appelées hémocytes chez les insectes), se déroule en deux vagues successives au cours du développement (pour revue voir $[10,11]$ ). 


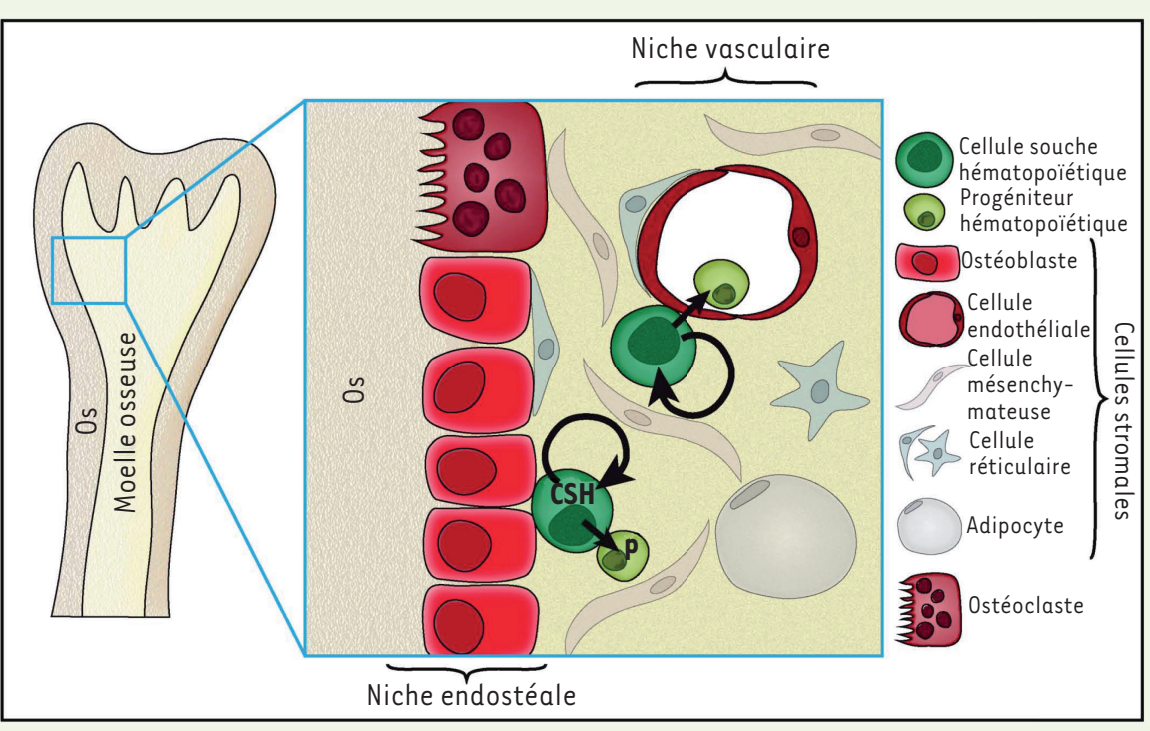

Figure 1. La niche hématopoïétique chez les mammifères. Chez les mammifères adultes, I'hématopoïèse a lieu dans la moelle osseuse. Les cellules souches hématopoïétiques (CSH, vert foncé) s'autorenouvellent (flèche) et donnent naissance aux progéniteurs hématopoïétiques ( $P$, vert clair). Les cellules composant la niche, appelées cellules stromales, correspondent à différents types cellulaires. Deux compartiments majeurs de la niche hématopoiétique ont été identifiés et sont étroitement imbriqués: la niche endostéale, majoritairement composée d'ostéoblastes, et la niche vasculaire, correspondant aux petits vaisseaux sanguins [33].

- La première vague a lieu au stade embryonnaire à partir du mésoderme antérieur et est à l'origine de la spécification de deux types d'hémocytes circulants, apparentés au lignage myéloïde des vertébrés. Les plasmatocytes, équivalents fonctionnels des macrophages, sont impliqués dans la phagocytose. Les cellules à cristaux, fonctionnellement apparentées aux plaquettes, sont requises pour la cicatrisation et la mélanisation, une réaction spécifique aux arthropodes.

- La seconde vague d'hématopoïèse a lieu au stade larvaire dans un organe hématopoïétique dédié, appelé glande lymphatique. Outre des plasmatocytes et des cellules à cristaux, un troisième type cellulaire, les lamellocytes, se différencient dans la glande lymphatique en réponse à un stress immun, tel que l'infection par des guêpes parasitoïdes, parasites naturels des drosophiles. Les lamellocytes sont de grandes cellules très adhérentes, qui encapsulent et neutralisent un pathogène trop gros pour être détruit par phagocytose. La glande lymphatique se désagrège au début de la métamorphose et ses cellules sont libérées dans la circulation [12]. Ainsi, au cours de la métamorphose et dans les mouches adultes, l'hémolymphe contient un mélange d'hémocytes d'origine embryonnaire et d'origine larvaire [13]. À ce jour, aucun processus hématopoïétique n'a été décrit au stade adulte.

\section{La glande lymphatique : l'organe hématopoïétique larvaire de la drosophile}

La glande lymphatique se forme au cours de l'embryogenèse, au contact du tube cardiaque qui est le système vasculaire de la mouche (Figure 2A). Au cours des trois stades larvaires successifs (durée totale de 96 h), la glande lymphatique augmente de taille. Au dernier stade larvaire, la glande est mature et les hémocytes se différencient. À ce stade, la glande est composée de deux lobes antérieurs (ou primaires) formés au stade embryonnaire, et d'une suite de plus petits lobes postérieurs (ou secondaires) formés au cours des stades larvaires [14]. Ces lobes secondaires correspondent à des réservoirs de progéniteurs hématopoïétiques (Figure $2 B$ ). Les lobes antérieurs comportent trois zones: la zone médullaire (ZM) qui contient les progéniteurs hématopoïétiques pluripotents; la zone corticale (ZC), localisée au cortex de la glande, où se différencient les hémocytes; et le PSC (posterior signaling center), un centre de signalisation localisé dans la partie postérieure et composé d'environ 30 cellules (Figure 2B) [15]. La spécification du PSC au cours de l'embryogenèse requiert la fonction du gène collier (col), qui code pour un facteur de transcription conservé au cours de l'évolution. Quatre gènes apparentés (orthologues) ont été identifiés chez les mammifères : les gènes early B-cell factor (ebf), dont deux, ebfl et ebf2 sont impliqués dans l'hématopoïèse [16-19]. L'analyse de mutants col a permis de montrer que le PSC est requis pour maintenir un pool de progéniteurs hématopoïétiques pluripotents dans la glande lymphatique $[20,21]$, une fonction similaire à celle de la niche des CSH chez les vertébrés. Le PSC de drosophile constitue donc un modèle de choix, complémentaire du modèle de la souris, pour étudier in vivo les mécanismes cellulaires et moléculaires impliqués dans la formation et la fonction de la niche hématopoïétique.

\section{Les voies de signalisation impliquées dans la fonction du PSC}

L'invalidation de gènes spécifiques du PSC ou de chacune des deux autres zones a montré que le PSC sécrète des signaux diffusibles, capables d'activer différentes voies de signalisation dans les autres compartiments de la glande lymphatique. Deux de ces signaux issus du PSC ont été bien caractérisés: le morphogène Hh 


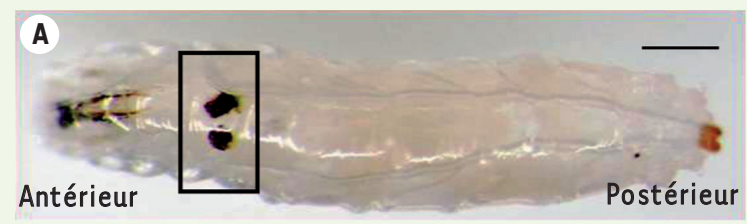

B Hémocytes différenciés (ZC)

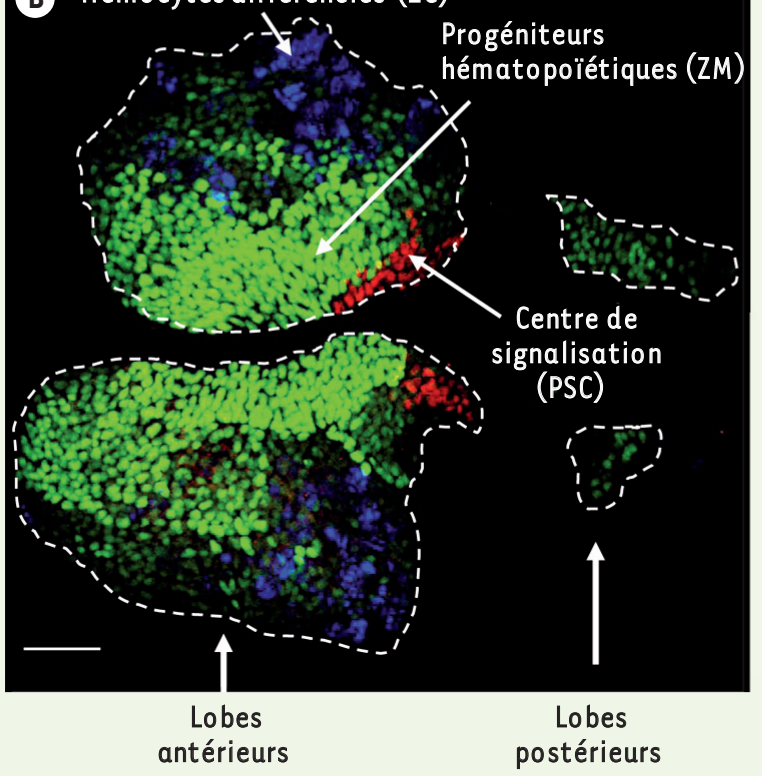

C

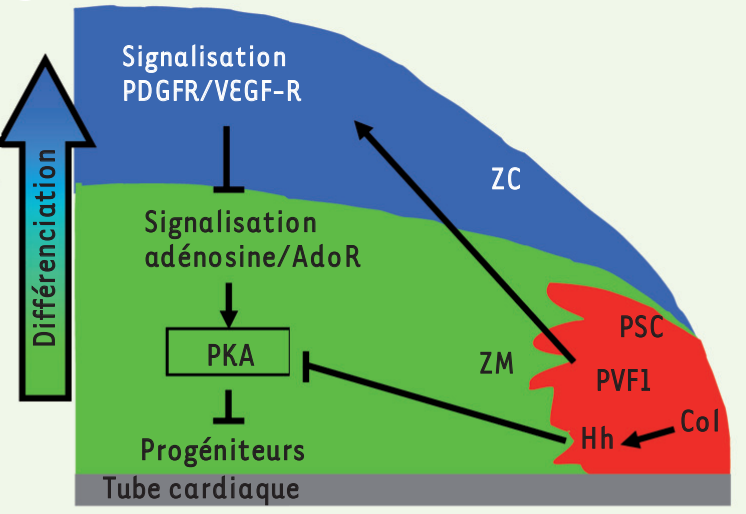

Figure 2. La niche hématopoïétique chez la drosophile. A. Image d'une larve mutante où la glande lymphatique nécrosée est marquée en noir. La barre d'échelle correspond à $0,5 \mathrm{~mm}$. B. Image en microscopie confocale d'une glande lymphatique de larve de drosophile (dernier stade larvaire). Les lobes antérieurs (ou primaires) sont les plus développés et sont organisés en trois zones. La zone médullaire (ZM, en vert) est composée de progéniteurs hématopoïétiques et la zone corticale ( $Z C$, en bleu) d’hémocytes différenciés provenant de la différenciation des progéniteurs. La troisième zone, le centre de signalisation (PSC, en rouge), est

formée par un petit groupe de cellules localisées au pôle postérieur du lobe antérieur. Les cellules du centre de signalisation (PSC, en rouge) expriment le facteur de transcription Collier (Col). Les lobes postérieurs (ou secondaires) sont composés de progéniteurs hématopoïétiques et servent de réservoirs de cellules. La barre d'échelle correspond à $80 \mu \mathrm{m}$. C. Représentation schématique de la partie postérieure d'un lobe antérieur. Les cellules du PSC (rouge) envoient des signaux requis pour le maintien des progéniteurs de la ZM. Collier (Col) contrôle l'expression du morphogène Hedgehog ( $\mathrm{Hh}$ ) dans le PSC. Le ligand Hh diffuse et active la voie de signalisation Hh dans la ZM, qui est nécessaire au maintien de l'état progéniteur via la répression de la protéine kinase A (PKA). La voie de signalisation PDGF/VEGFR est activée dans la ZC grâce à la diffusion du ligand PVFl synthétisé dans le PSC. L'activation de cette voie conduit à l'inactivation de la voie de signalisation adénosine/AdoR dans la ZM. Cette dernière contrôle positivement l'activité de PKA.

(hedgehog) et le ligand PVFl (platelet-derived growth factor/vascular endothelial growth factor-like factor) (Figure 2C). La perte de fonction dans le PSC des gènes hh ou pvfl conduit à la différenciation prématurée des progéniteurs hématopoïétiques. Cependant, les cibles et communications cellulaires de ces deux signaux sont très différentes. Hh agit directement sur les progéniteurs de la ZM et est nécessaire au maintien de leur état progéniteur pluripotent [21]. PVF] diffuse par exocytose à partir du PSC et agit, via son récepteur PDGF/ VEGFR, dans les hémocytes en cours de différenciation. II y stimule la sécrétion d'ADGF-A (adenosine deaminase-related growth factor-A), une enzyme modifiant l'adénosine extracellulaire. Cette modification conduit à l'inactivation de la voie de signalisation adénosine/AdoR (adenosine receptor) dans les cellules de la ZM. La voie AdoR régule positivement l'activité de la protéine kinase A (PKA), favorisant la différenciation, alors que la signalisation Hh inhibe l'activité PKA dans la ZM. Le contrôle positif de l'activité PKA par les cellules se différenciant, via la voie adénosine/AdoR, et négatif par le PSC, via la voie Hh, permet un maintien de l'équilibre entre progéniteurs et cellules différenciées [22]. D'autres études ont montré que la voie JAK (Janus kinase)/STAT (signal transducer and activator of transcription) est activée et nécessaire dans la ZM [23]. Ces données montrent que les communications entre le PSC et la ZM sont indispensables au maintien de l'équilibre entre cellules progéniteurs pluripotentes et cellules différenciées au sein de la glande lymphatique, et mettent en jeu de multiples voies de signalisation.

\section{La voie de signalisation BMP/Dpp contrôle le nombre de cellules de la niche hématopoïétique chez la drosophile}

La voie de signalisation BMP (bone morphogenetic protein), conservée au cours de l'évolution, est impliquée 


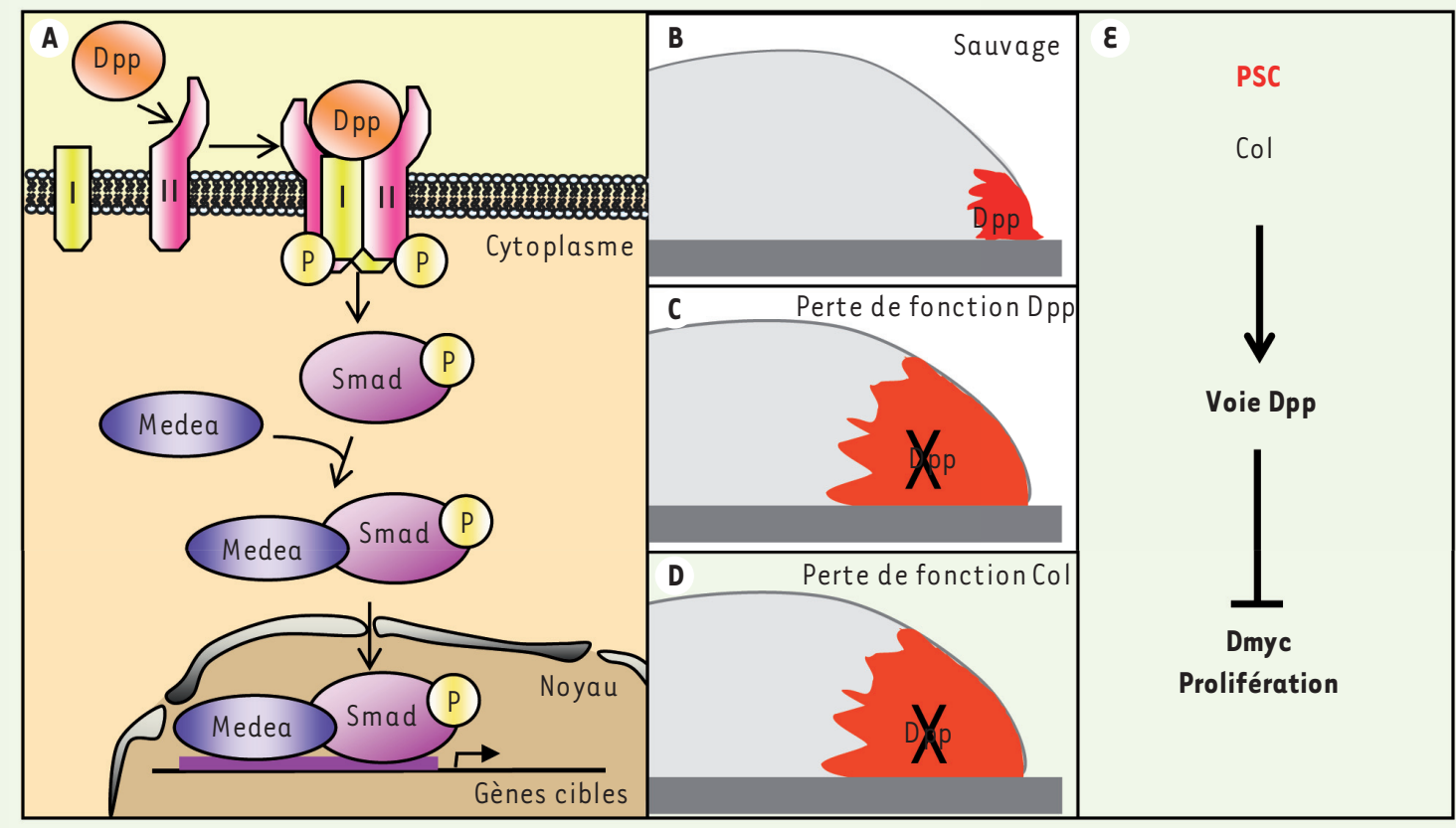

Figure 3. La voie de signalisation BMP/Dpp contrôle la taille du PSC. A. Le ligand Dpp se fixe sur le récepteur de type II, conduisant à la formation d'un complexe composé de récepteurs de type I et de type II. Ce complexe activé s'autophosphoryle et phosphoryle le facteur de transcription Smad. Smad phosphorylé recrute son cofacteur (Medea) et entre dans le noyau pour activer la transcription de gènes cibles. B. Au stade larvaire, la voie BMP/Dpp est activée dans le PSC (en rouge). L'inactivation dans le PSC de cette voie $\left(\boldsymbol{C}_{\text {. }}\right)$ ou de col ( $\boldsymbol{D}_{\text {. }}$ ) conduit à une augmentation massive du nombre de cellules composant le PSC. La perte de fonction de Col dans le PSC ( $\boldsymbol{D}$.) entraîne une inactivation de la voie BMP/Dpp dans ces cellules indiquant que Col contrôle l'activation de cette voie. $\varepsilon$. Représentation schématique de la cascade de régulation contrôlant le nombre de cellules du PSC. L'activation de la voie BMP/Dpp par Collier dans le PSC réprime l'expression du proto-oncogène Myc (Dmyc chez la drosophile), impliqué dans la prolifération.

dans de nombreux processus biologiques [24-26]. Cette voie est activée suite à la liaison d'un ligand de type TGF $\beta$ (transforming growth factor $\beta$ ) à un récepteur de type II, suivie du recrutement et de la phosphorylation d'un récepteur de type I. Le complexe récepteur type I plus II phosphorylé active par phosphorylation un facteur de transcription Smad (mothers against decapentaplegic homolog). Smad activé recrute un cofacteur, Smad4/Medea, puis devient nucléaire et active la transcription de ses gènes cibles (Figure 3A). Chez la drosophile, la voie BMP, appelée Dpp (decapentaplegic), comporte trois ligands (Dpp, glass bottom boat et screw), deux récepteurs de type I (thickvein [Tkv] et saxophone), deux récepteurs de type II (wishfull thinking [Wit] et Punt), un transactivateur Smad et son cofacteur Medea [25]. Au sein de la glande lymphatique, la voie Dpp est spécifiquement activée dans le PSC par liaison de Dpp à ses récepteurs Tkv et Wit [27]. L'inactivation génétique de la signalisation Dpp dans le PSC conduit à une augmentation considérable du nombre de cellules du PSC, montrant que la voie Dpp contrôle la prolifération des cellules du PSC (Figure 3B). Ce contrôle de la prolifération passe par la répression de l'expression de Dmyc, l'orthologue chez la drosophile du protooncogène Myc vertébré (Figure $3 \xi$ ). Il a été montré indépendamment que la voie de signalisation wingless (Wg/Wnt) [28] régule positivement le nombre de cellules du PSC, suggérant que le rapport entre les niveaux de signalisation Wnt et Dpp détermine la taille du PSC [27, 29]. L'étape suivante était de déterminer les conséquences du changement du nombre de cellules du PSC sur la différenciation des hémocytes (Figure 4A) [21, 27].

\section{L'homéostasie des cellules au sein de la glande lymphatique est dépendante de la taille du PSC}

L'inactivation de la signalisation Dpp dans le PSC induit à la fois une augmentation du nombre des cellules du PSC et une perte de différenciation des hémocytes, indiquant que le contrôle de la taille du PSC est un paramètre essentiel de l'homéostasie hémocytaire dans la glande lymphatique (Figure 4A). L'expression de Hh n'est cependant pas affectée dans ces conditions [21], ce qui suggère que l'augmentation du nombre de cellules du PSC provoque une augmentation de la quantité de Hh total produit, qui conduit au maintien de toutes les cellules de la LG (hors PSC) à l'état de progéniteurs multipotents [27]. Une perte de fonction de col dans le PSC à partir du deuxième 


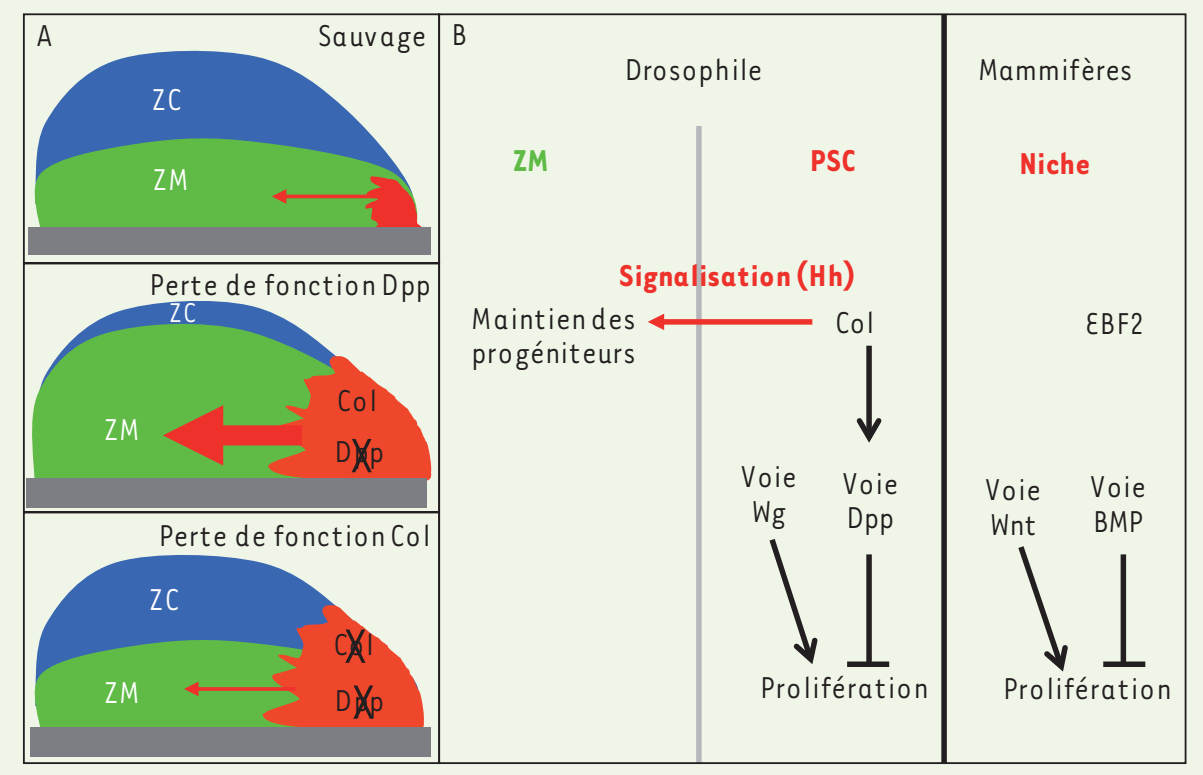

Figure 4. Contrôle de la taille et de la fonction de la niche hématopoiétique de drosophile: nouveaux parallèles avec les mammifères. A. L'inactivation de la voie BMP/Dpp dans le PSC entraîne une augmentation du nombre de cellules du PSC et, par conséquent, du niveau des signaux émis par celui-ci. Ceci conduit préférentiellement au maintien des progéniteurs de la ZM au détriment des hémocytes différenciés. L'inactivation de col dans le PSC conduit également à une augmentation du nombre de cellules du PSC. Cependant, la balance entre la ZM et la ZC n'est pas modifiée par rapport au contexte sauvage. $\boldsymbol{B}$. Chez la

drosophile, Col contrôle à la fois l'expression du signal Hh et l'activation de la voie de signalisation BMP/Dpp. La signalisation Wg contrôle également le nombre de cellules de la niche. Cette étude révèle de nouveaux parallèles entre les rôles de Col, BMP/Dpp et Wg chez la drosophile et leurs orthologues mammifères EBF2, BMP et Wnt, dans le contrôle de l'hématopoïèse.

stade larvaire, après la phase de spécification dans l'embryon, conduit à une augmentation du nombre de cellules du PSC. Cette augmentation est corrélée à la perte d'activation de la voie BMP dans le PSC. Pourtant, contrairement à la simple inactivation de la voie Dpp, l'augmentation de la taille du PSC consécutive à l'absence de Col n'empêche pas la différenciation des hémocytes. D'où l'hypothèse que l'augmentation du nombre de cellules du PSC, en absence de Col, est compensée par une perte partielle de leur activité de signalisation (Figure 4). En accord avec cette hypothèse, l'expression de Hh dans les cellules du PSC est significativement diminuée dans des conditions de perte de fonction de Col. L'ensemble de ces données montre que le facteur de transcription Collier coordonne les programmes de prolifération et de signalisation des cellules de la niche hématopoïétique, et que cette coordination est un paramètre essentiel de l'hématopoïèse (Figure $4 B$ ).

\section{De la drosophile aux mammifères}

Bien que les données concernant la formation et la fonction de la niche hématopoïétique chez la drosophile et la souris restent fragmentaires, de nombreux parallèles peuvent déjà être établis entre ces deux organismes modèles. Ainsi, chez la souris, la perte de fonction du gène codant pour la $\beta$-caténine, facteur de transcription en aval de la voie Wg/Wnt, entraîne une diminution du nombre d'ostéoblastes [30]. Un phénotype comparable est observé chez la drosophile où l'inactivation du ligand Wg dans le PSC conduit à la réduction de la taille de ce dernier (Figure 4B) [27, 29]. Un second parallèle concerne l'implication de la voie BMP/Dpp requise pour le contrôle de la taille de la niche chez la drosophile. En effet, l'inactivation d'un récepteur de la voie de signalisation BMP (BMPRIA) dans les ostéoblastes conduit à une augmentation de leur nombre et, ainsi, du nombre de CSH [31]. Enfin, de nouveaux parallèles pourraient être établis sachant qu'EBF2 (early B-cell factor 2), I'un des orthologues murins de Col, est exprimé dans les ostéoblastes et est requis pour le maintien des CSH [32]. De la même manière, chez la drosophile, Col est requis pour le maintien des précurseurs hématopoïétiques. Cependant, le mécanisme impliqué demeure non élucidé et les interactions possibles entre EBF2 et les voies BMP et Wnt restent à établir. De même, une meilleure connaissance des voies de signalisation impliquées dans la communication entre la niche et les CSH chez les mammifères [33] permettra de déterminer si les voies de signalisation Hh et PDGF/VEGFR (platelet derived growth factor) vascular endothelial growth factor), impliquées dans ce processus chez la drosophile, jouent aussi un rôle dans la niche des vertébrés. $\diamond$

\section{SUMMARY}

The drosophila hematopoietic niche

Stem cells are required for both tissue renewal and repair in response to injury. The maintenance and function of stem cells is controlled by their specific cellular microenvironment called "niche". Hematopoietic stem cells (HSC) that give rise to all blood cell types have 
been extensively studied in mammals. Genetic and molecular analyses performed in mice identified several signaling pathways involved in the cellular communications between HSC and their niche. However, hematopoietic niche plasticity remains poorly understood. The discovery of a Drosophila hematopoietic niche, called PSC, established a new model to decipher the niche function in vivo. Size control of the PSC is essential to maintain hematopoietic tissue homeostasis and a molecular cascade controlling the PSC cell number has been characterized. Novel parallels between Drosophila and mammalian hematopoietic niches open new perspectives for studies of HSC biology in human. $\diamond$

\section{REMERCIEMENTS}

Nous remercions L. Bataillé, J.L. Frendo, M. Meister et A. Vincent pour la lecture et la correction du manuscrit. Ce travail de recherche est financé par le CNRS, I'Université Toulouse III, le ministère de la Recherche (ANR-11 BSV2 00801), I'ARC (Association pour la recherche sur le cancer), la FRM (Fondation pour la recherche médicale ; DEQ 20090515429) et une bourse ARC pour I. Morin-Poulard.

\section{LIENS D’INTÉRÊT}

Les auteurs déclarent n'avoir aucun lien d'intérêt concernant les données publiées dans cet article.

\section{RÉFÉRENCES}

1. Trumpp A, Essers M, Wilson A. Awakening dormant haematopoietic stem cells. Nat Rev Immunol $2010 ; 10: 201-9$.

2. Morrison SJ, Spradling AC. Stem cells and niches: mechanisms that promote stem cell maintenance throughout life. Cell 2008 ; 132 : 598-611.

3. Levesque JP, Helwani FM, Winkler IG. The endosteal «osteoblastic » niche and its role in hematopoietic stem cell homing and mobilization. Leukemia $2010 ; 24$ : 1979-92.

4. Lymperi S, Ferraro F, Scadden DT. The HSC niche concept has turned 31 . Has our knowledge matured? Ann NY Acad Sci $2010 ; 1192: 12-8$.

5. Mercier FE, Ragu C, Scadden DT. The bone marrow at the crossroads of blood and immunity. Nat Rev Immunol $2012 ; 12: 49-60$.

6. Lataillade JJ, Brunet de la Grange P, Uzan G, Le Bousse-Kerdilès MC. Les cellules souches ont-elles l'âge de leur niche? Med Sci (Paris) $2010 ; 26: 582-5$.

7. Schofield R. The relationship between the spleen colony-forming cell and the haemopoietic stem cell. Blood Cells $1978 ; 4: 7-25$.

8. Martinez-Agosto JA, Mikkola HK, Hartenstein V, Banerjee U. The hematopoietic stem cell and its niche: a comparative view. Genes Dev 2007 ; 21 : 3044-60.

9. Andriatsilavo M, Gervais L, Fons C, Bardin AJ. L'intestin moyen de drosophile. Un nouveau modèle d'étude des cellules souches in vivo. Med Sci (Paris) $2013 ; 29: 75-81$.

10. Crozatier M, Meister M. Drosophila haematopoiesis. Cell Microbiol 2007 ; 9 : 1117-26.

11. Lanot R, Zachary D, Holder F, Meister M. Postembryonic hematopoiesis in Drosophila. Dev Biol $2001 ; 230: 243-57$.

12. Grigorian M, Mandal L, Hartenstein V. Hematopoiesis at the onset of metamorphosis: terminal differentiation and dissociation of the Drosophila lymph gland. Dev Genes Evol 2011 ; 221 : 121-31.

13. Holz A, Bossinger B, Strasser T, et al. The two origins of hemocytes in Drosophila. Development $2003 ; 130: 4955-62$.
14. Krzemien J, Crozatier M, Vincent A. Ontogeny of the Drosophila larval hematopoietic organ, hemocyte homeostasis and the dedicated cellular immune response to parasitism. Int J Dev Biol 2010 ; 54 : 1117-25.

15. Jung SH, Evans CJ, Uemura C, Banerjee U. The Drosophila lymph gland as a developmental model of hematopoiesis. Development 2005; 132:2521-33.

16. Wang MM, Reed RR. Molecular cloning of the olfactory neuronal transcription factor Olf-1 by genetic selection in yeast. Nature $1993 ; 364$ : 121-6.

17. Hagman J, Belanger C, Travis A, et al. Cloning and functional characterization of early B-cell factor, a regulator of lymphocyte-specific gene expression. Genes Dev $1993 ; 7: 760-73$.

18. Hagman J, Gutch MJ, Lin H, Grosschedl R. EBF contains a novel zinc coordination motif and multiple dimerization and transcriptional activation domains. EMBO J 1995 ; 14 : 2907-16.

19. Daburon V, Mella S, Plouhinec JL, et al. The metazoan history of the COE transcription factors. Selection of a variant HLH motif by mandatory inclusion of a duplicated exon in vertebrates. BMC Evol Biol $2008 ; 8$ : 131.

20. Krzemien J, Dubois L, Makki R, et al. Control of blood cell homeostasis in Drosophila larvae by the posterior signalling centre. Nature 2007 ; 446 : 325-8.

21. Mandal L, Martinez-Agosto JA, Evans CJ, et al. A Hedgehog- and Antennapedia-dependent niche maintains Drosophila haematopoietic precursors. Nature $2007 ; 446: 320-4$

22. Mondal BC, Mukherjee T, Mandal L, et al. Interaction between differentiating cell- and niche-derived signals in hematopoietic progenitor maintenance. Cell $2011 ; 147: 1589-600$.

23. Makki R, Meister M, Pennetier D, et al. A short receptor downregulates JAK/ STAT signalling to control the Drosophila cellular immune response. PLOS Biol $2010 ; 8$ : el 1000441.

24. Harris RE, Ashe HL. Cease and desist: modulating short-range Dpp signalling in the stem-cell niche. EMBO Rep $2011 ; 12: 519-26$.

25. Affolter $M$, Basler $K$. The decapentaplegic morphogen gradient: from pattern formation to growth regulation. Nat Rev Genet $2007 ; 8: 663-74$.

26. Sagorny K, Chapellier M, Laperrousaz B, Maguer-Satta V. BMP et cancer : le Yin et le Yang des cellules souches. Med Sci (Paris) $2012 ; 28: 416-22$.

27. Pennetier D, Oyallon J, Morin-Poulard I, et al. Size control of the Drosophila hematopoietic niche by bone morphogenetic protein signaling reveals parallels with mammals. Proc Natl Acad Sci USA 2012 ; 109 : 3389-94.

28. Gilgenkrantz H. Le monde selon YAP. Un dialogue perpétuel entre les voies Wnt et Hippo. Med Sci (Paris) 2013 ; 29 : 868-74.

29. Sinenko SA, Mandal L, Martinez-Agosto JA, Banerjee U. Dual role of wingless signaling in stem-like hematopoietic precursor maintenance in Drosophila. Dev Cell 2009; $16: 756-63$.

30. Nemeth MJ, Mak KK, Yang Y, Bodine DM. Beta-catenin expression in the bone marrow microenvironment is required for long-term maintenance of primitive hematopoietic cells. Stem Cells $2009 ; 27$ : 1109-19.

31. Zhang J, Niu C, Ye L, et al. Identification of the haematopoietic stem cell niche and control of the niche size. Nature $2003 ; 425: 836-41$.

32. Kieslinger $M$, Hiechinger $S$, Dobreva $G$, et al. Early B cell factor 2 regulates hematopoietic stem cell homeostasis in a cell-nonautonomous manner. Cell Stem Cell $2010 ; 7: 496-507$.

33. Wilson A, Laurenti $\varepsilon$, Trumpp A. Balancing dormant and self-renewing hematopoietic stem cells. Curr Opin Genet Dev 2009 ; 19 : 461-8.
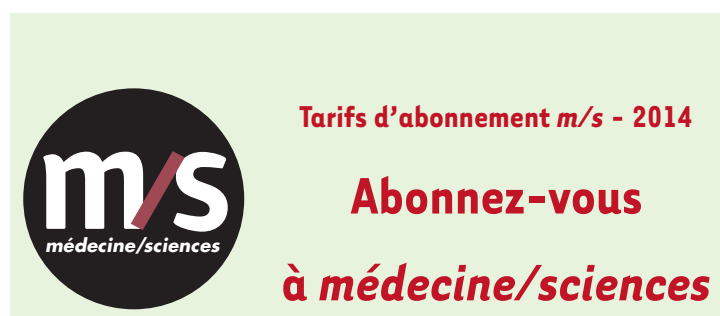

$>$ Grâce à $m / s$, vivez en direct les progrès des sciences biologiques et médicales

\section{TIRÉS À PART}

M. Crozatier 